\title{
Doppler effect in zero-index metamaterials
}

\author{
$\underline{\text { Jia Ran }}{ }^{1,2}$, Yewen Zhang ${ }^{1}$, Xiaodong Chen ${ }^{2,3}$, Kai Fang ${ }^{1}$, Hong Chen ${ }^{1}$ \\ ${ }^{1}$ Tongji University, School of Physics Science and Engineering, Siping Road, 200092, Shanghai, China \\ ${ }^{2}$ Queen Mary University of London, School of Electronic Engineering and Computer Science, Mile End, E1 \\ 4NS, London, UK \\ 2University of Electronic Science and Technology of China, Chengdu, 610054, China. \\ ranjia121@126.com
}

\begin{abstract}
This paper presents our observation of the abnormal Doppler effect inside a reconfigurable balanced transmission line, which has zero index at the balanced frequency. By applying a digital signal controller, a moving reflective interface is built inside this transmission line. The normal, inverse and zero Doppler effects are observed at different frequencies, which gives a completed picture of Doppler effect in metamaterials.
\end{abstract}

\section{INTRODUCTION}

The Doppler effect is the shift of wave frequency when there is a relative movement between the source and observer, due to the change of the successive distance between the wave fronts. It has been well established in normal materials (Double positive materials, DPG) and has many applications in astronomy, radar and metrology. With the development of metamaterials [1-3], the inverse Doppler effect has also attracted avid interest in double negative metamaterials (DNG), that is, the frequency will increase when the source is leaving away from the detector while decrease when it is moving towards the detector [4-6].

Inspired by the recent research on the zero-index metamaterials (ZIM) [7], here comes the idea to study the Doppler effect in the zero-index metamaterials. The wave amplitude inside the ZIM keeps the same in space but vibrates in time [8], i.e. it has no wave front. Therefore there would be no change of successive distance between the wave fronts when there is a relative movement between the source and the observer. This new abnormal phenomenon is different from the inverse Doppler effect, which predicts a reversed frequency shift compared to what the normal Doppler effect expects.

The zero index metamaterial is achieved based on a balanced composite right/left-handed transmission line (CRLH TL) loaded with parallel varactors. Each transmission line unit shows a passband (band gap) by applying high (low) level bias voltages. By changing the bias voltages from low level to high level in succession, a moving reflective interface can be built inside the transmission line and a relative movement between the source and the spectrum analyzer is realized as a result, shown in Fig. 1(a).

\section{THE CHARACTERISTICS OF THE RECONFIGURABLE TRANSMISSION LINE}

The CRLH TL unit is fabricated on the FR4 dielectric slab with relative permittivity 4.75 , composed of two $15 \mathrm{pF}$ series capacitors, one $10 \mathrm{nH}$ parallel inductor and a parallel NXP BB131 varactor, displayed in Fig. 1(b). The band gap will shut down with $15 \mathrm{pF}$ series capacitance and $16 \mathrm{~V}$ bias voltages, meanwhile the transmission line turns to be balanced. The red curves in Fig. 1(c) represents the balanced dispersion whose relative permittivity and permeability are both near zero at the zero-index frequency $f_{Z}=852 \mathrm{MHz}$. Frequencies under $f_{Z}$ are set in the left-handed passband while in the right-handed passband when beyond $f_{Z}$.

The capacitance of the varactor will vary within the bias voltage, leading to the variation of the dispersion line. A wideband gap would appear when turn off the bias voltage, as the dotted line in Fig. 1(d) shows. Therefore, By altering the bias voltages from $0 \mathrm{~V}(16 \mathrm{~V})$ to $16 \mathrm{~V}(0 \mathrm{~V})$ continuously and injecting the wave from the left (right) port, a (an) receding (approaching) reflective interface is formed and the Doppler effect in DNG/ZIM/DPG can be observed by choosing incident frequency under/at/beyond the zero-index frequency. 


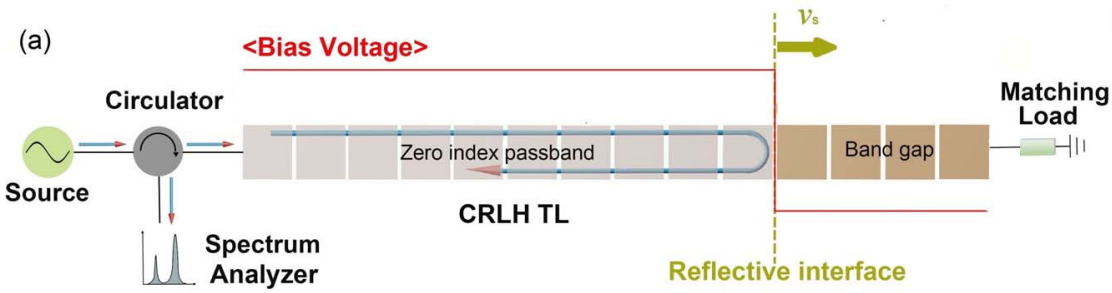

(b)

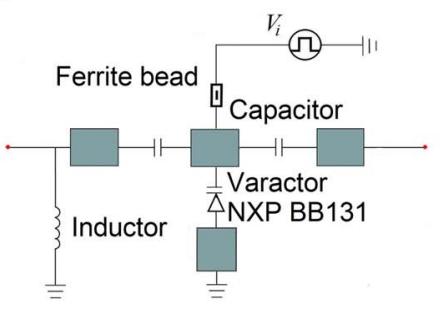

(c)

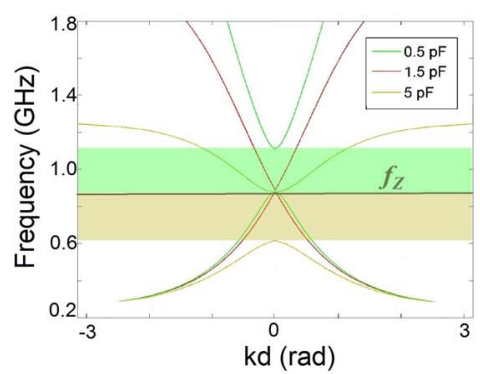

(d)

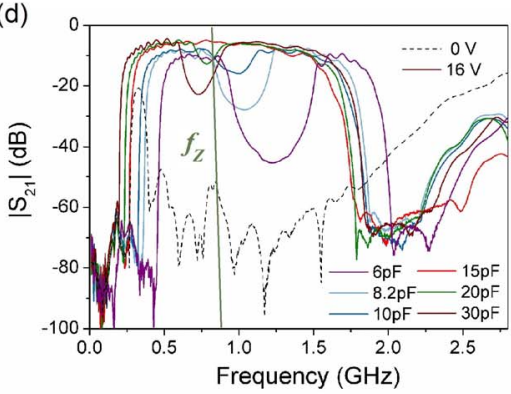

Fig. 1. (a) Schematic of the system to realize the Doppler effect in the zero index metamaterials. The red line is the bias voltage level applied to the corresponding transmission line unit; (b) Schematic of the transmission line unit. $V_{i}(i=1,2 \ldots 14)$ are the bias voltages applied to the $i$ th unit; (c) Dispersion lines of the transmission line with different series capacitors, applied $16 \mathrm{~V}$ bias voltage; (d) Measured $\left|S_{21}\right|$ of the transmission line under $0 \mathrm{~V}$ (dotted line) and $16 \mathrm{~V}$ (solid lines), loaded with different series capacitors.

\section{DESIGN OF THE DIGITAL SIGNAL CONTROLLER AND THE MEASURED DOPPLER EFFECTS}

The digital signal controller consists of a cascade of 14 edge-triggered JK flip-flops and 14 monostable multivibrators, shown in Fig. 2(a) (only two units are shown here for simplicity). As Fig. 2(b) displays, the negative going transition (NGT) of the clock signal enables the positive going transition (PGT) of the output $Q$ under the condition that the trigger signal is at high level. The output $\bar{Q}$ of the previous flip-flop is connected to the input $\mathrm{K}$ of the next flip-flop, which leads to that the delay time of each outputs' PGT is controllable by the clock signal period $\tau$. As a result, the moving velocity of the reflective interface can be dominated by the clock signal period as $v_{s}=d / \tau$, where $d$ is the length of the TL unit.

The shortest period $\tau$ available in our experiment is $200 \mathrm{~ns}$, giving rise to the fastest velocity of the reflective interface $v_{s}=62 \mathrm{~km} / \mathrm{s}$. The velocity is positive when the interface is receding from the source. Under this circumstance, the Doppler effect in the zero-index metamaterials is carried out, as Fig. 2(e) shows, accompanied with the normal and inverse Doppler effects in Fig. 2(d) and (f) separately. The strong reflection at the incident frequency on the spectrum is due to the impedance mismatch at the input port of the transmission line. wave is due to The incident frequency $700 \mathrm{MHz}$ in Fig. 2(d) is set in the left-handed passband, the Doppler frequency is lower than the incident frequency when the interface is approaching the source, as the inverse Doppler effect predicts, while since $980 \mathrm{MHz}$ is set in the right-handed passband, the Doppler effect indicates that the Doppler frequency would be higher than the operating frequency, as Fig. 2(f) shows, vice versa.

Different from what normal and inverse Doppler effects describe, there is no extra peak (Doppler frequency) either when the interface is approaching or receding from the source if the incident frequency is the zero-index frequency, shown in Fig. 2(e). On the other words, there is no Doppler shift in the zero-index metamaterials. Owing to the unique characteristic of zero-index metamaterials that the amplitude of wave inside the ZIM keeps the same in the space, there would be no change of successive distance between the wave fronts no matter how the relative movement between the source and observer is, which is the causation of the Doppler effect in both normal and negative-index materials. As a result, the Doppler frequency cannot be generated no matter how the interface moves. 
(a)

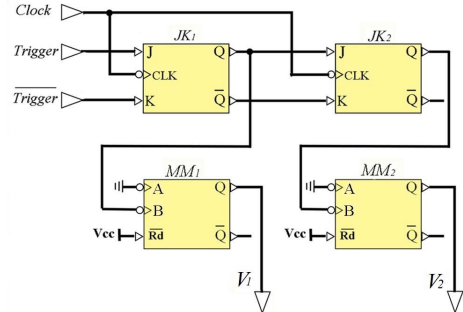

(b)

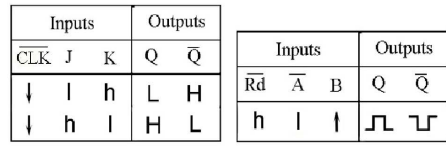

(c)

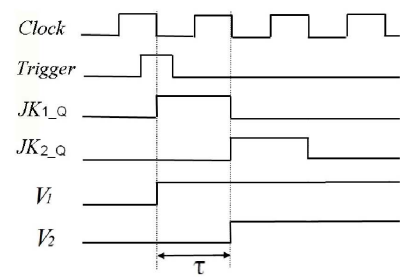

$(\mathrm{d})$ (e)
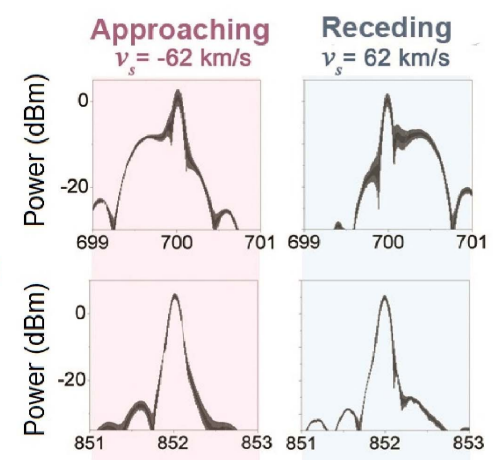

(f)

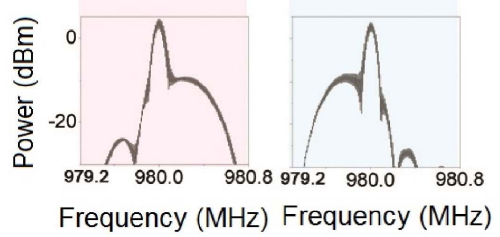

Fig. 2. (a) Schematic of two units of the digital signal controller; (b) Truth tables of the JK flip-flop (left) and monostable multivibrator (right); (c) Timing diagram of the main outputs in (a); Spectra of the reflected signals with incident frequency $700 \mathrm{MHz}$ (d), $852 \mathrm{MHz}$ (e) and $980 \mathrm{MHz}$ (f), when the moving velocity of the reflective interface are $-62 \mathrm{~km} / \mathrm{s}$ (left column) and $62 \mathrm{~km} / \mathrm{s}$ (right column).

\section{CONCLUSION}

By choosing the proper incident frequency that exhibits a zero-index behavior inside the CRLH transmission line loaded with parallel varactors, the Doppler effect inside the ZIM is observed, accompanied with the normal and inverse Doppler effects. Contrasting to the two known Doppler effects, the wave in the ZIM exhibits an abnormal performance that there is no Doppler frequency in the wave reflected by the moving interface. This unusual phenomenon may trigger potential applications in zero-index metamaterials.

\section{ACKNOWLEDGEMENT}

This work is supported by the National Basic Research Program (973) of China (No.2011CB922001), National Natural Science Foundation of China (No.11234010) and China Scholarship Council.

\section{REFERENCES}

[1] J. B. Pendry, "Negative refraction makes a perfect lens," Phys. Rev. Lett., vol. 85, p. 3966, 2000.

[2] D. R. Smith, W. J. Padilla, D. C. Vier, S. C. Nemat-Nasser, S. Schultz, "Composite medium with simultaneously negative permeability and permittivity," Phys. Rev. Lett., vol. 84, p. 4184, 2000.

[3] R. A. Shelby, D. R. Smith, S. Schultz, "Experimental verification of a negative index of refraction," Science, vol. 292, p. 77-79, 2001.

[4] N. Seddon, T. Bearpark, "Observation of the inverse Doppler effect," Science, vol. 302, p. 1537-1540, 2003.

[5] J. Ran et al., "Realizing tunable inverse and normal Doppler shifts in reconfigurable RF metamaterials," Sci. Rep., vol. 5 , no. 11659,2015

[6] J. B. Chen et al., "Observation of the inverse Doppler effect in negative-index materials at optical frequencies," Nature Photon. Vol. 5, p. 239-242, 2011.

[7] X. Huang, Y. Lai, Z. H. Hang, H. Zheng, C. T. Chan, “ Dirac cones induced by accidental degeneracy in photonic crystals and zero-refractive-index materials," Nat. Mater., vol. 10, p. 582-586, 2011.

[8] R. W. Ziolkowski, "Propagation in and scattering from a matched metamaterial having a zero index of refraction," Phys. Rev. E, vol. 70, p. 046608, 2004. 